\title{
Insights about Large-Scale Online Peer Assessment from an analysis of an Astronomy MOOC
}

\begin{abstract}
In this work we investigate the peer grading assignments which were an integral part of the astronomy Massive Open Online Course (MOOC) "Astronomy: Exploring Time and Space" provided through Coursera from March to May 2015. Our general goal is to assess the role of peer graded assignments in such courses and how they contribute to students' learning and motivation. In order to achieve this broad goal we look at the peer grading process from multiple perspectives. We present an analysis of demographics for peer grading participants and show how they are different from the general course population. We also look at different aspects of peer grading assignments such as lengths of essays, time spent grading, number of gradings performed, final grades and percentage of relevant videos watched. We compare these distributions for different assignments and also their correlations on a level of individual learners. We show that participation in the first peer graded assignment is the best predictor of completion for the course as a whole. Moreover, learners who did well on the first peer graded assignment show better engagement and do better in the course overall. Finally, we report on validity and reliability of peer graders as compared to instructor graders and trained undergraduate graders.
\end{abstract}

Keywords: Peer Assessment, MOOCs, Online Learning, Informal Learning

\section{Introduction}

As the name suggests, Massive Open Online Courses (MOOCs) are universitylevel courses which are accessible online and open to all learners for free or for a small fee if they wish to earn a completion certificate. The "massive" in the name

Preprint submitted to Computers \& Education

June 6, 2018 
5 refers to number of people enrolled in such classes which is typically on the order of tens of thousands. MOOCs gained a lot of public awarness at the beginning of this decade when the main providers, Coursera, Udacity and edX, launched their platforms. The year 2012 was even proclaimed "the year of the MOOC" by The New York Times (Pappano, 2012). Despite their very enthusiastic onset, the debate about what is going to be the role of MOOCs in our society is still very much alive (Hollands \& Tirthali, 2014; MacAndrew \& Scanlon, 2013). It is still not clear whether MOOCs are a cost-effective solution for providing higher level education to masses and how effectively they support learning.

There are scores of online educational videos and course materials that can be readily found on almost any topic on platforms such as YouTube, iTunes U, MIT OpenCourseWare. What distinguishes MOOCs from them is the fully integrated assessment in the course design. Assessment and especially formative assessment transforms the learner interaction from passive reading and listening to a true learning experience that engages learners with the studied material (Suen, 2014). Since the inception of MOOCs, course designers have experimented with various forms of assessment (Sandeen, 2013; Kearns, 2012). To successfully employ an assessment in the online setting, we should be able to:

- Make the assessment scalable: Any assessment we wish to use has to be effective even if the course is taken by tens of thousands of people.

- Verify identity of the users: We have to make sure that the person taking the course is the same as the person who undertook the assessment. For example Coursera is using automated approaches such as typing pattern recognition or photo identification (Maas et al., 2014). Other approaches such as using video proctors where the learner is monitored through a web camera for the duration of the exam impose significant costs $(\$ 15-\$ 45$ per session plus cost of the equipment) on the learner.

- Make it simple to use: The MOOC interface has to be simple enough so that technological challenges do not distract from learning and do not impede from achieving the learning goals. 
- Manage learner time and workload: MOOC audience comes from multiple countries and time zones, so it is not feasible to have face to face meetings of big cohorts of learners, therefore assessment has to be asynchronous.

- Provide feedback: It is desirable for the instructor to address common issues and concerns in the update post or utilize Q \& A type discussion boards.

There are two main options for assessments satisfying all the criteria above. One is automated machine grading and the other is a peer grading. Automated grading is eminently suitable for grading quizzes, computer codes and calcula45 tions. It can be used whenever the outcome of the assignment is well defined, quantitative and predictable. Carefully designed machine grading can be also formative and provide valuable feedback to the learner. There have been experiments with automated grading of written assignments (Balfour, 2014), typically for scoring essays in English writing classes, but most of the time peer grading is used. The other option is peer grading which employs MOOC participants to evaluate the work of several of their colleagues and provide them with feedback.

When considering peer grading the two main criteria which determine its usefulness are validity (Hunter, 1983) and reliability (Shrout \& Fleiss, 1979). Reliability measures the consistency of multiple graders when asked to grade 55 the same assignment and validity indicates how much the peer graders differ from an ideal score assigned by an expert grader.

Suen (2014) argues that there are multiple sources of inconsistencies in peer grading situations, arising from different interpretation of rubrics, variations in grader competence, tendencies to be either lenient or stringent, and also conditions influencing the grader such as his surroundings, emotional and physical state. We can hope to eliminate most of these by constructing a good rubric and by training graders carefully. As was shown in a review by Panadero \& Jonsson (2013) a well design rubric can positively influence learning and learners typically have a positive perception of rubrics (Reddy \& Andrade, 2010). Most 
importantly use of rubrics was proven to improve the reliability of the grades (Jonsson \& Svingby, 2007). All studies known to the authors that asses validity of a peer review process compare grades assigned by learners to a single instructor grade on a selected subset of assignments. Therefore, in this work we would like to see what is the level of disagreement among different kinds of expert 70 graders, and how does it compare with peer graders.

\section{Peer grading literature review}

In this section we present literature on the peer grading as it historically evolved from traditional classes to blended or online classes and ultimately to MOOCs.

The term "peer grading" is defined by Falchikov (1995) as the process through which groups of individuals rate their peers. Even early-on researchers attributed to peer gradings other uses than just pure grading mechanism (Dochy et al., 1999). For example Somervell (1993) argues that peer grading is a learning process on its own which develops useful reflection skills and Keaten \& Richardson (1993) suggest that peer assessment encourages high levels of responsibility among students. Williams (1992) discovered that the majority of students perceive benefits of peer grading especially in comparison of approaches, in comparison of standards and in exchange of information.

There are many studies on the validity and reliability of peer grading in traditional classes and the results are mainly favorable. A review of 31 peer grading studies by Topping (1998) marked only 7 examples of peer grading assignments to have unacceptably low validity or reliability. A similar meta study by Falchikov \& Goldfinch (2000) of 48 studies (from which 30 were different than Topping's) identifies variables which are influencing the validity of peer grading such as global vs. compartmentalized rubric, design of the assessment, familiarity with the subject, and nature of the delirevable in question.

Shortcomings of peer grading are abundant in literature as well. Cheng \& Warren (1997) found out about the necessity to properly and systematically train the 
peer graders how to assess their peers. Pond et al. (1995) reports undesirable tendencies arising in traditional classroom settings such as friendship markings (inflated grade for friends). On the other hand Sadler \& Good (2006) points out a tendency to award lower grade to the best performing students in the middle school science class settings.

Peer assessment has a more natural place in online and blended classes because the logistical aspect of distributing assignments for grading, anonymizing authors and graders, providing and reviewing feedback and computation of the final grade can be handled by technology. There have been several studies of this problem on a level of individual courses (Bouzidi \& Jaillet, 2009; Tseng \& Tsai, 2007), but to our best knowledge, there are no comparative studies across multiple subjects and course designs.

Peer grading turned out to be instrumental in Massive Open Online Courses, the scale of which makes it one of the few viable options for assessment. Intelligence is required to find evidence of student learning from their work which is at the limit of current automated systems (O'Toole, 2013).

It is economical to use peer graders compared to hired expert graders, but peer grading may be perceived by both students and faculty as less credible. The main reasons are that there is very little instructor supervision, student graders are novices in the field and not necessarily motivated to take the peer assessment seriously. Also because of the random nature of assigning graders, the quality of feedback and consistency in grading can vary greatly from assignment to assignment. A recent study of MOOC instructors carried out by The Chronicle of Higher Education showed that $74 \%$ used some sort of automated grading and $34 \%$ of respondents used peer grading (Kolowich, 2013). While $67 \%$ of them considered automated grading very reliable only $26 \%$ of respondents thought the same about peer grading. Participants of an experiment to improve engineering reports writing skills through peer review (Furman \& Robinson, 2003) showed concerns about usefullness of the peer grading process. $56 \%$ of respondents considered peer review to be somewhat or very helpful and $44 \%$ not helpful. But when asked if they would recommend using it again in the future only 
$17 \%$ agreed. Overall $60 \%$ were strongly against it and the remaining $23 \%$ were undecided. The mixed opinion of the learners on the usefulness of the peer review is also apparent on the MOOC level as reported by Meek et al. (2016).

Suen (2014) summarized the various attempts that have been made to improve peer grading process. For example, Calibrated Peer Review ${ }^{\mathrm{TM}}$ evaluates 30 the reliability of each grader on a well known sample of test assignments for which correct grades are provided by an instructor. So it is possible to determine credibility of each grader and weight the grades they assigned accordingly. Another approach is to use statistical methods, such as Bayesian post hoc stabilization, to correct grade distributions of the whole class after the grading is finished (Goldin \& Ashlev, 2011).

Our study contributes to this literature in determining overall impact of the peer grading assignment by connecting the performance of the peer grading participants with other aspects of the course.

\section{Research questions}

The overall goal for this study was to determine the value of peer graded assignments in MOOCs. In particular we wanted to understand the relationship between participation in the peer graded assignments and student motivation and learning. The research questions guiding this study were as follows

1. How does participation in a peer graded assignment predict learner success and completion?

2. Who participates in a peer graded assignment? Are they any demographic differences from the rest of the learners?

3. Were there any noticeable changes between individual peer graded assignments throughout the course session?

4. Are learners valid and reliable peer graders and is it possible to improve their validity and reliability by training them?

5. What are the relationships between grades and student participation in various aspects of the peer graded writing assignment. 


\section{Research Settings, Methodology and Design}

155 our research together with the data sources and methology of their processing.

\subsection{Research Settings}

In this work we evaluate a peer assessment for an astronomy MOOC called "Astronomy: Exploring Time and Space", which was offered by Coursera from March to May 2015. The aim of this MOOC was to provide the general public with a broad introduction to topics in astronomy which are currently undergoing rapid research development. The course curriculum covered topics like exploration of the Solar System, exoplanet detection, the construction of large telescopes, progress in cosmology and prospects of detecting extraterrestrial life.

The content of the course was delivered primarily through 18 hours of video lectures divided into short (on average 8 - 12 minute) segments. Other supplemental materials, such as lecture slides and a free online textbook accessible through instructor's web site "Teach Astronomy" (Impey et al., 2013), were provided. This course was session-based, meaning, that all students progressed through the course at the same pace imposed by releases of new modules at a weekly basis and assignments deadlines.

Out of 25,379 people who registered for the course, 14,900 actually accessed the website at least once, 12,042 watched at least one video lecture, 4275 completed at least one graded assignment and 1607 of them ultimately earned a passing grade of $50 \%$ or more. 1109 of graduates completed the course with "distinction" by scoring higher than 80\%. People from 151 different countries participated in the course, making the audience truly global. Around $34 \%$ of them came from the United States followed by India (8\%), UK (5\%) Canada (4\%) China (4\%) and Brazil (3\%). The participants turned out to be highly educated with only $16 \%$ not having any university experience. In the age distribution we saw that 50\% of learners belonged into age cathegory 21-40 years old. Only $13 \%$ were younger and the rest - $37 \%$ were older. Finally, the male to 
female ratio was approximately $3: 2$. If you would like to learn more about our MOOC users we recently published an in-depth study of the learner demographics, engagement, motivations and predictors of completion for this particular course (Impey et al., 2016).

The assessment consisted of 13 automatically graded quizzes from the lecture material worth $60 \%$ of the grade, three peer graded written assignments worth $20 \%$ and two online activities involving real observational data worth remaining $20 \%$.

\subsection{Data sources and analysis}

The demographic information of our MOOC learners rely on two sources: geographic location was determined from the IP address of the Coursera users and the rest of demographic data i.e. age, gender, highest education and occupation was obtained from an external Entry Survey administered through Survey Monkey to the self selected sample of 2,465 participants. In order to find out the previous level of academic experience with astronomy and familiarity of learners with the subject we used two single response questions from another Entry Survey which was administered by Coursera with 4,969 participants.

The comprehensive data about all aspects of the peer grading assignments have been made available to us by Coursera. The processing was done using non parametric statistic, specifically Kruskal-Wallis H test and Dunn's post hoc test to compare distributions of dependent variables for different assignments.

In order to investigate validity and reliability of peer graders we had a sample of 300 essays graded by a group of three course instructors and by trained undergraduate graders who were either majors or minors in Astronomy. Each essay from this sample was graded by all three instructors and by three randomly chosen trained undergraduate graders from a group of 7 people. Reliability was tested using the Intraclass Correlation Coefficient (ICC) (Shrout \& Fleiss, 1979) and validity was investigated based on the correlation with the median of instructors' grades and their deviations from the median. 


\subsection{Peer Graded Assignments and Process}

For each of the three peer graded assignments we provided three writing prompts and the topics were: figures of merit for telescopes across the electromagnetic spectrum, the detection of exoplanets and their properties, and the nature of exotic end states of stars (Appendix A). Students were asked to formulate their response within 7 days using no more than 500 words. This limit on the length of submission was not enforced. Technical aspect of the grading was handled by Coursera. Each assignment was graded by at least three randomly selected peers using a global 0 - 4 points scale rubric (Appendix B). Comparative study by Falchikov \& Goldfinch (2000) showed that a rubric based on a global judgment of the assignment is more valid in the peer grading settings.

The final grade for the assignment was the median of grades received from the peer graders. Students participating in the peer grading were supposed to review submissions of four of their colleagues in the course. If they failed to do that for any reason their final grade from this particular assignment was reduced by $20 \%$. As we will show later, the percentage of learners who did not do the grading is quite high and that is the reason why we have essays with only three grades.

\section{Findings}

The following five sub-sections of this section address each research question respectively. The first sub-section characterizes course completition rates of peer grading participants. The second sub-section quantifies the demographic characteristics of peer grading participants. The third one compares all three peer graded assignments. The fourth one investigates the reliability and validity of peer graders. Finally the last sub-section looks for the relationships between grades and student participation in various aspects of the peer graded writing assignment. 


\subsection{Course Completion Rates for Peer Grading Participants}

240 the best predictor of completion for the course as a whole which answers the first research question. About $81 \%$ of the learners who submitted their first essay successfully graduated from the course. This number improves to $89 \%$ for learners who did all the required grading. To put these high numbers into per245 spective, only $11 \%$ of those who opened the course graduated. For comparison other predictors of completion were:

- watching the first video (14\% graduated),

- participation in the external entry survey (33\% graduated),

- and completing the first quiz (36\% graduated) (Impey et al., 2016).

\subsection{Demographics of Peer Grading Participants}

In this paragraph we address the second research question - whether learners participating in the peer graded assignments are any different from the general population of the course. First of all we looked on geographic data of our learners. We had access to IP addresses of all learners which we used to determine their geographical locations. There were no significant differences between learners who wrote three essays and those who did all required gradings, but when compared with all learners who at least opened the course, there was a noticeable drop in percentage share of peer graders from Asia (by 40\%) and similar increase in the percentage share of peer graders from Europe (Figure 1).

We also investigated other demographic indicators, as presented in Figure 2 and 3. These data were obtained from the initial external survey $(\mathrm{n}=2,465)$ which our learners were asked to complete. As a consequence we do not have demographic information for all peer review participants, so we can only make assumptions about the learners who participated in both the external survey and

(out of 945) and for learners who did all required gradings 401 learners (out of 679). From the data in Figure 2 and 3 we infer that compared to the learners 


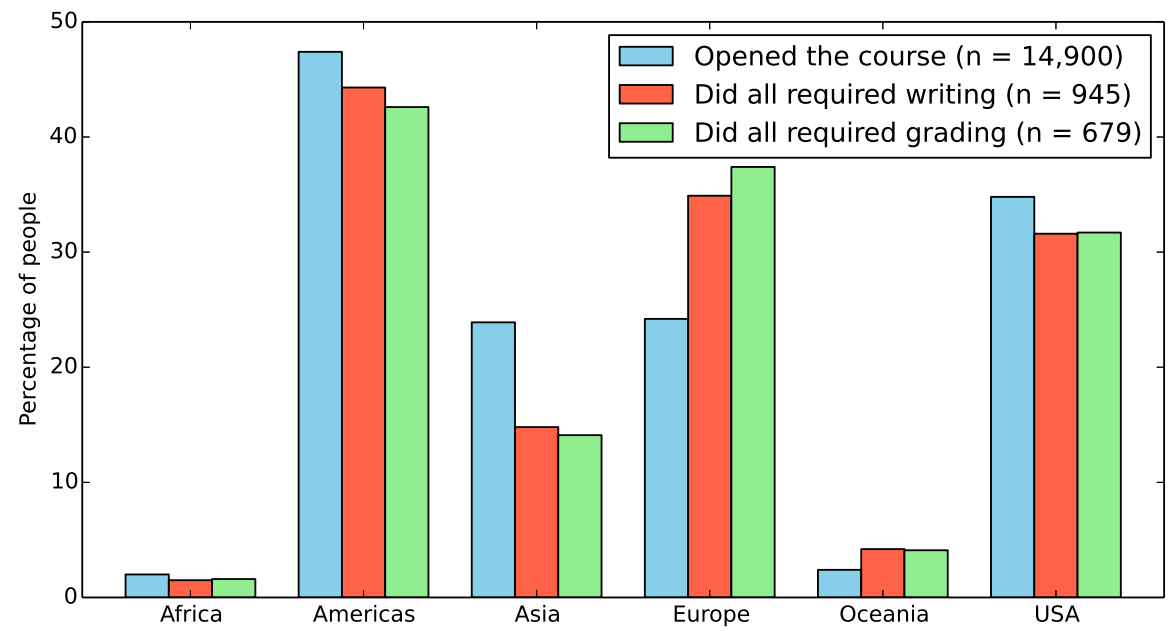

Figure 1: Comparison of geographical distributions for learners who participated in the peer graded assignments with general population of the course.

who took the external survey, participants in the peer graded assignments were more likely male, older, and more educated. People working in professional fields (science/engineering), technical fields (software/computers), educators, and those who were retired were more likely to participate in the peer grading assignments. Surprisingly, learners who listed as their occupation "Student" were much less likely to write essays (by 26\%) and grade other learners (by $42 \%$ ). Notice remarkable similarity with the results of a similar study of peer grading in a biomedical science MOOC (Meek et al., 2016). Other self reported occupations as working at home, in service/retail, administrative, law, medicine or unemployed learners did not show any noticeable differences and are not included in Figure 3b

Finally, we were interested in the level of previous astronomy knowledge of our learners. This question was addressed in the Coursera entry survey $(\mathrm{n}=$ 4,969). This survey almost completely samples the peer grading participants. To be specific out of 945 learners who submitted all essays, 922 answered and out of the 679 learners who did all required grading, 668 participated in this survey. We can see that over $60 \%$ of peer grading participants didn't have any 


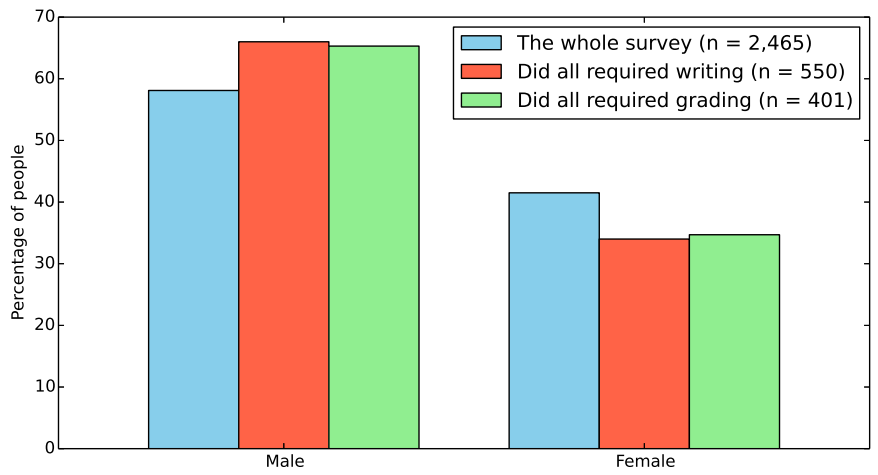

(a) Gender of participants

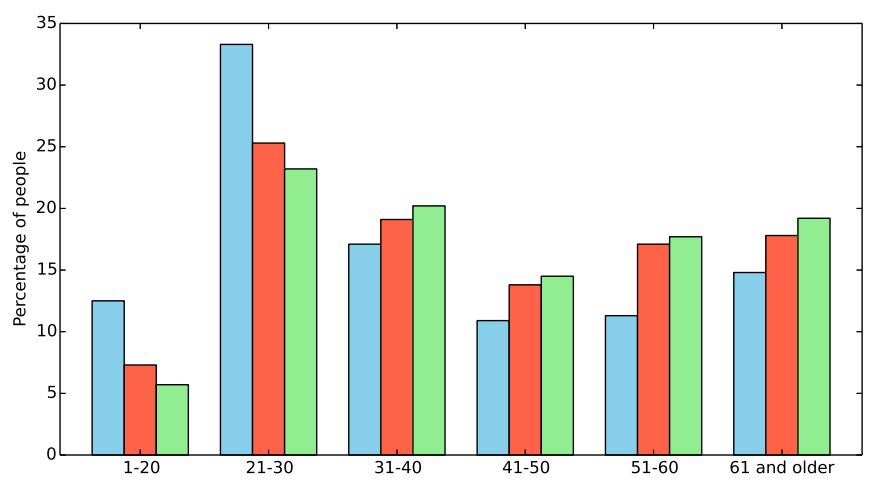

(b) Age of participants

Figure 2: Comparison of demographic distributions for learners who participated in the peer graded assignments with learners who participated in the external entry survey. 


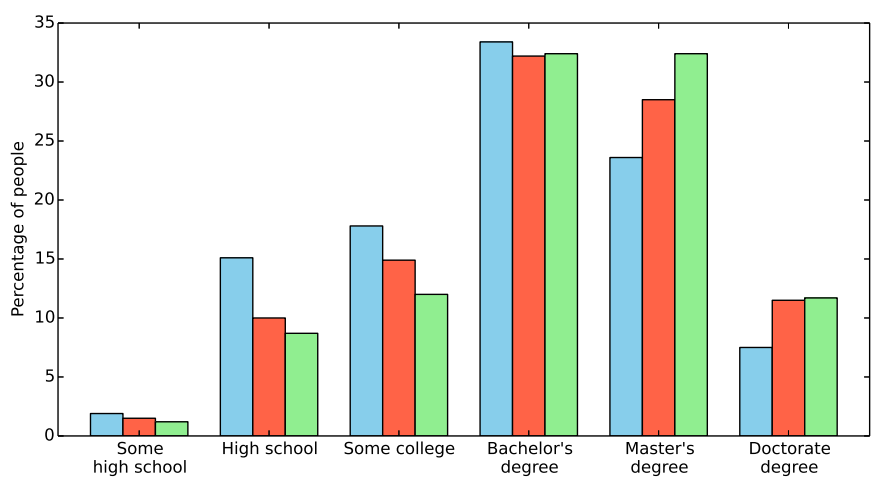

(a) Highest education of participants

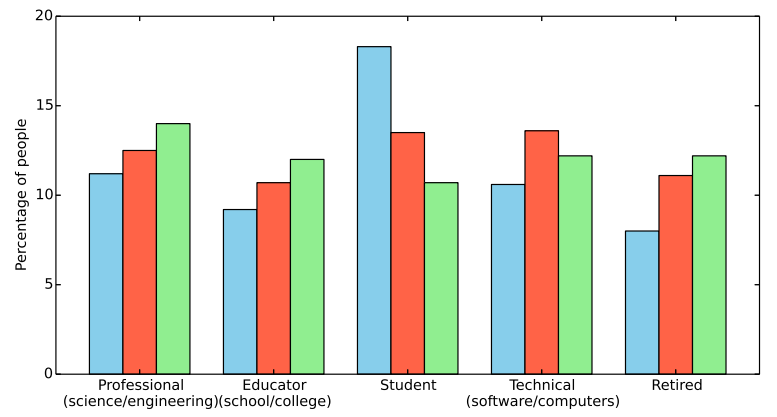

(b) Occupations of participants

Figure 3: Comparison of demographic distributions for learners who participated in the peer graded assignments with learners who participated in the external entry survey. 


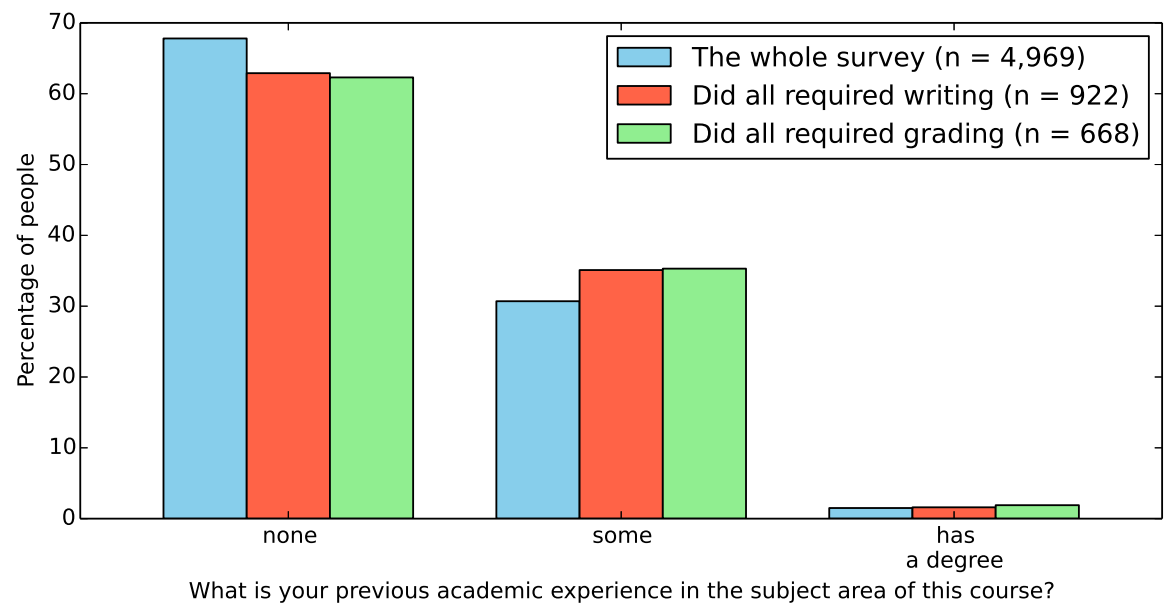

Figure 4: The level of previous academic experience in Astronomy based on the Coursera entry survey.

previous academic experience in Astronomy (Figure 4) and over $80 \%$ of learners were not familiar or somewhat familiar with the subject itself (Figure 5 ).

\subsection{Characteristics of the Peer Graded Assignments}

This sub-section looks at different aspects of peer graded assignments, namely how many learners participated, the length of essays submitted, what were the grades they received and gave, how many reviews they performed, how much time they spent on average grading one essay, and finally, how many relevant instructional videos they watched. We also compared these distributions for all three assignments and looked on differences in order to answer the third research question.

The number of learners who submitted their work was 1364 for the first assignment, 1241 for the second, and 1114 for the last one. Of those totals, $1064(78 \%), 1043(84 \%)$, and $926(83 \%)$ respectively, did the required number of four peer gradings or more (Figure [6). Overall, 1550 individual learners submitted at least one assignment and 1322 of them finished a peer grading review for at least one assignment. Finally, 945 learners submitted all three 


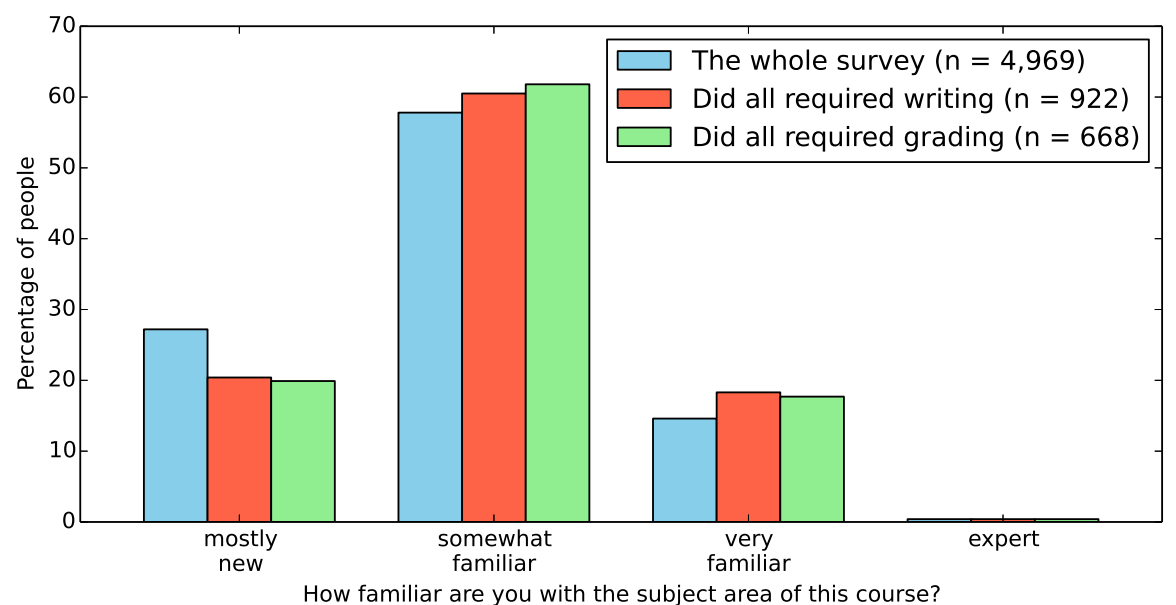

Figure 5: The level of familiarity with the subject of Astronomy based on the Coursera entry survey.

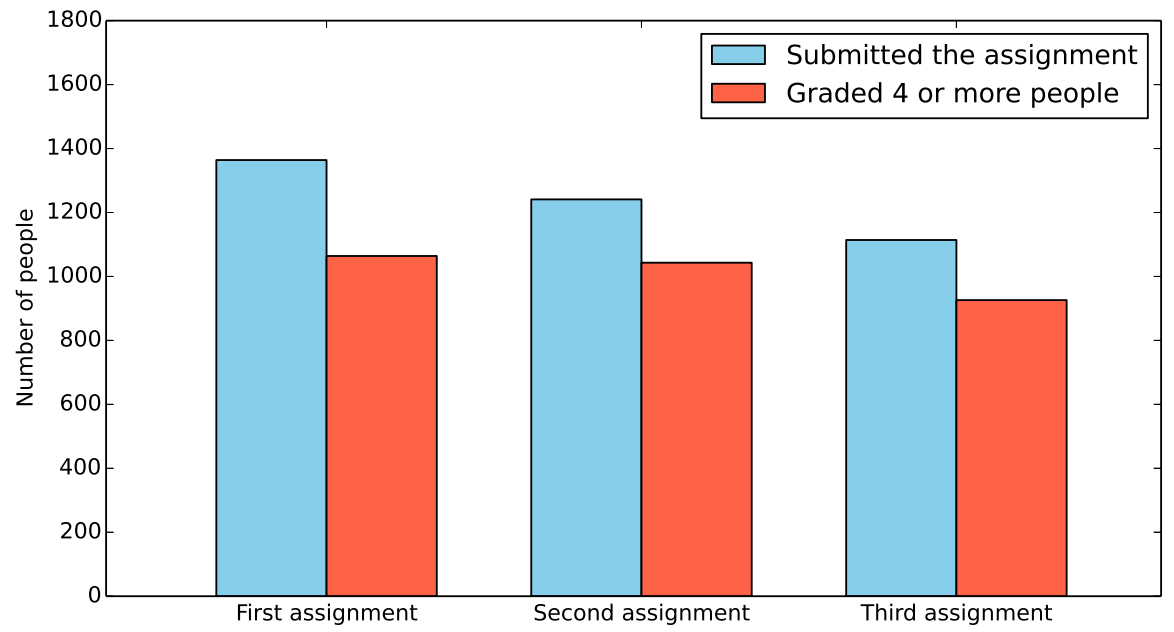

Figure 6: Number of learners who submitted each writing assignment (blue) and who graded the required number of other learners (red). Participation in peer grading for the three assignments is thus $78.0 \%, 84.0 \%$ and $83.1 \%$ respectively. 


\begin{tabular}{|c|c|c|c|c|}
\hline \multirow{2}{*}{$\begin{array}{c}\text { Assignment } \\
\text { number }\end{array}$} & \multicolumn{2}{|c|}{ Word count } & \multicolumn{2}{|c|}{ Essay grades (out of 4 ) } \\
\hline & Mean & St. Dev. & Mean & St. Dev \\
\hline $1 \mathrm{st}$ & $318^{*}$ & 146 & 3.31 & 0.75 \\
\hline 2nd & $353^{*}$ & 132 & 3.37 & 0.73 \\
\hline $3 \mathrm{rd}$ & $400^{*}$ & 118 & 3.23 & 0.86 \\
\hline Assignment & \multicolumn{2}{|c|}{ Count of peer gradings } & \multicolumn{2}{|c|}{ Avg. time spent grading (mins) } \\
\hline number & Mean & St. Dev. & Mean & St. Dev \\
\hline $1 \mathrm{st}$ & $4.49^{*}$ & 6.53 & $3.2^{*}$ & 2.5 \\
\hline 2nd & 3.83 & 2.62 & 4.6 & 3.0 \\
\hline $3 \mathrm{rd}$ & 3.79 & 2.80 & 4.8 & 3.0 \\
\hline $\begin{array}{c}\text { Assignment } \\
\text { number }\end{array}$ & $\begin{array}{l}\text { Fractic } \\
\text { Mean }\end{array}$ & Fraction of videos watched & & \\
\hline $1 \mathrm{st}$ & $87 \% *$ & $29 \%$ & & \\
\hline 2nd & $90 \%$ & $27 \%$ & & \\
\hline $3 \mathrm{rd}$ & $92 \%$ & $25 \%$ & & \\
\hline
\end{tabular}

Table 1: Comparison of descriptive statistics for all three peer grading assignments. Asterisks denote assignments which were statistically significantly different from the others for a given variable based on Dunn's post hoc test.

writing assignments and 679 of them completed all required peer gradings. The descriptive statistics for all assignments are available in Table 1 ,

The number of words in each assignment is plotted in Figure 7 A KruskalWallis $\mathrm{H}$ test showed that these distributions were statistically significantly different $\left(\chi^{2}(2)=205.85, p<0.001\right)$ and Dunn's post hoc test revealed that all assignments were different from each other, i.e. learners wrote on average progresively longer essays $(p<0.001)$. Although we imposed a limit of 500 words on each submission, a nontrivial number of learners wrote longer essays (12\% $23 \%)$.

Distribution of final grades after adjustment on peer grading participation described above can be found in Figure 8 . These distributions were not statisti- 


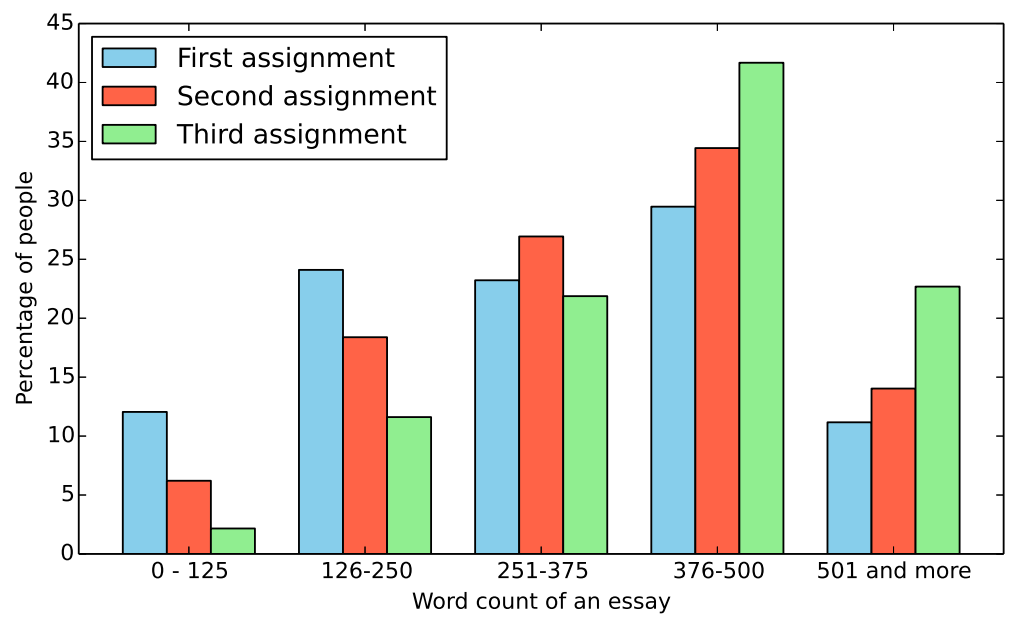

Figure 7: Distribution of length of essays in words for all three assignments.

cally significantly different according to the Kruskal-Wallis H test. $(p>0.001)$

Most learners who participated in the peer grading did exactly the required number of four reviews (Figure 9). To be specific, 44\%, 67\%, and $67 \%$ for each assignment. However, a surprisingly large number of learners did not grade at all: $21 \%, 15 \%$, and $16 \%$ respectively. There was a similar number of overachievers who graded more than 4 other essays: $34 \%, 17 \%$, and $16 \%$. As Figure 9 indicates the vast majority of these overachievers graded between 5 to 10 assignments, but we encountered a couple of outliers who graded as many as 130 assignments. This time Kruskal-Wallis $\mathrm{H}$ test showed that these distributions were statistically significantly different $\left(\chi^{2}(2)=34.18, p<0.001\right)$ and Dunn's post hoc test proved that the 1st assignment was different, i.e. learners on average did more reviews for the 1st assignment $(p<0.001)$. The difference between the number of gradings for the 2 nd and the 3rd assignment was not significant $(p=0.38)$.

From the data provided by Coursera, we were able to determine how much time each user spent on grading each essay. Coursera logs times of the first access to the essay by the grader and times of the grade submission. For each 


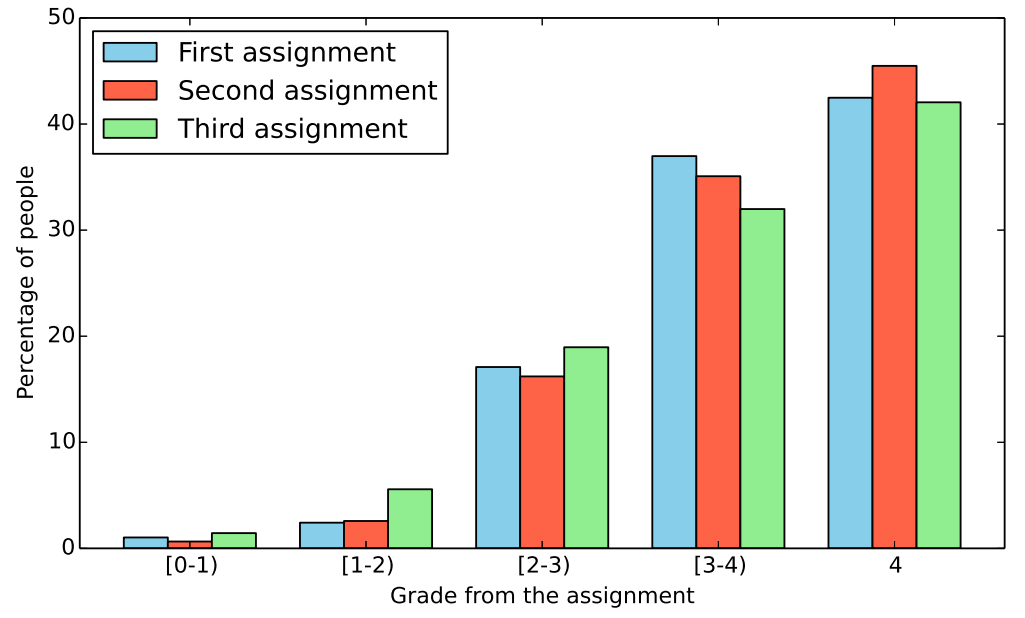

Figure 8: Distribution of final grades for all three peer-graded assignments.

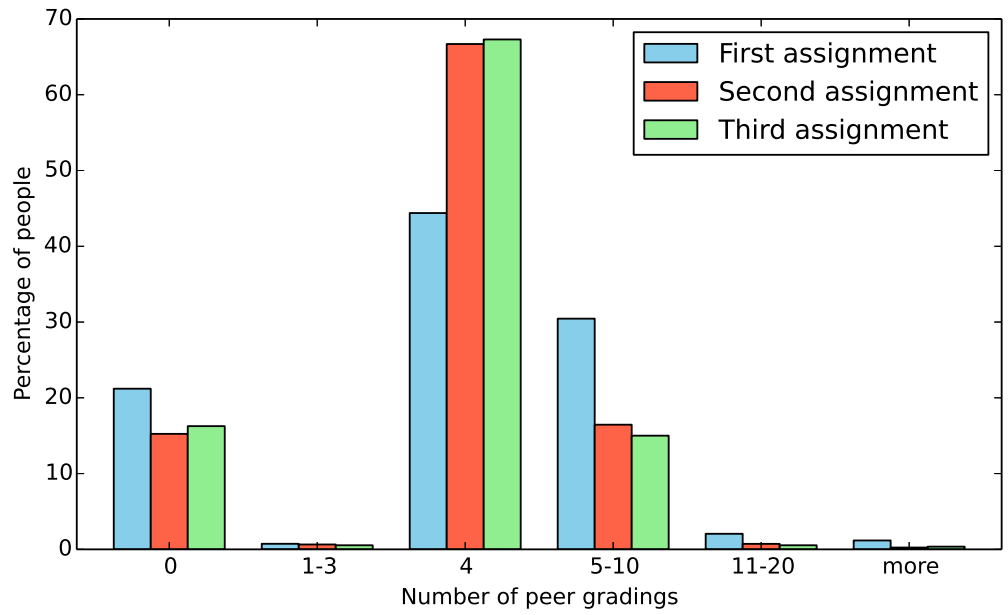

Figure 9: Number of learners who did certain number of peer gradings for each assignment 


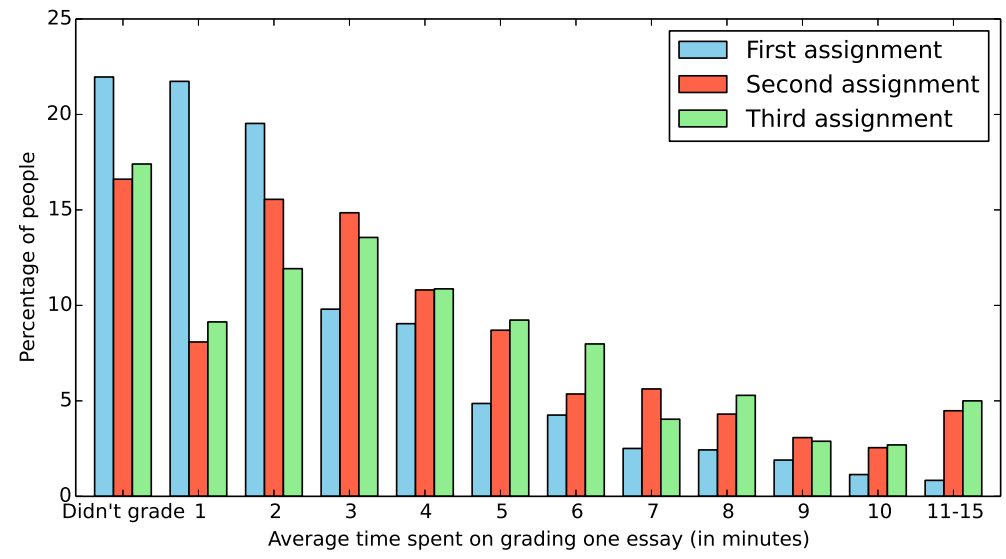

Figure 10: Distribution of average time spent on grading a single essay for each assignment. Times longer than 15 minutes were not included in this analysis.

assignment we averaged these times for each grader individually while discarding times longer than 15 minutes (Figure 10). We choose cut off of 15 minutes because after that time it is safe to assume that graders were no longer on a task. For the purposes of the analysis we omitted learners who did not grade at all (and thus their time is zero). The distributions were statistically significantly different as confirmed by a Kruskal-Wallis $\mathrm{H}$ test $\left(\chi^{2}(2)=172.46, p<0.001\right)$. Dunn's post hoc test showed that the 1st assignment was different, i.e. learners spent on average less time grading per essay for the 1st assignment $(p<0.001)$. The difference between the time taken to grade the 2 nd and the 3rd assignment was not significant $(p=0.52)$.

We also looked at how many instructional videos learners who submitted their assignment watched in the given week (Figure 11) and the result turned out to be fairly consistent across all three assignments. Kruskal-Wallis $\mathrm{H}$ test found that the difference between all three assignments is significant $\left(\chi^{2}(2)=32.04\right.$, $p<0.001$ ), and according to Dunn's post hoc test, the first assignment turned out different. In other words, learners watched on average fewer instructional videos for the first assignment than the others $(p<0.001)$. The difference between instructional videos watched for the 2nd and 3rd assignment was not 


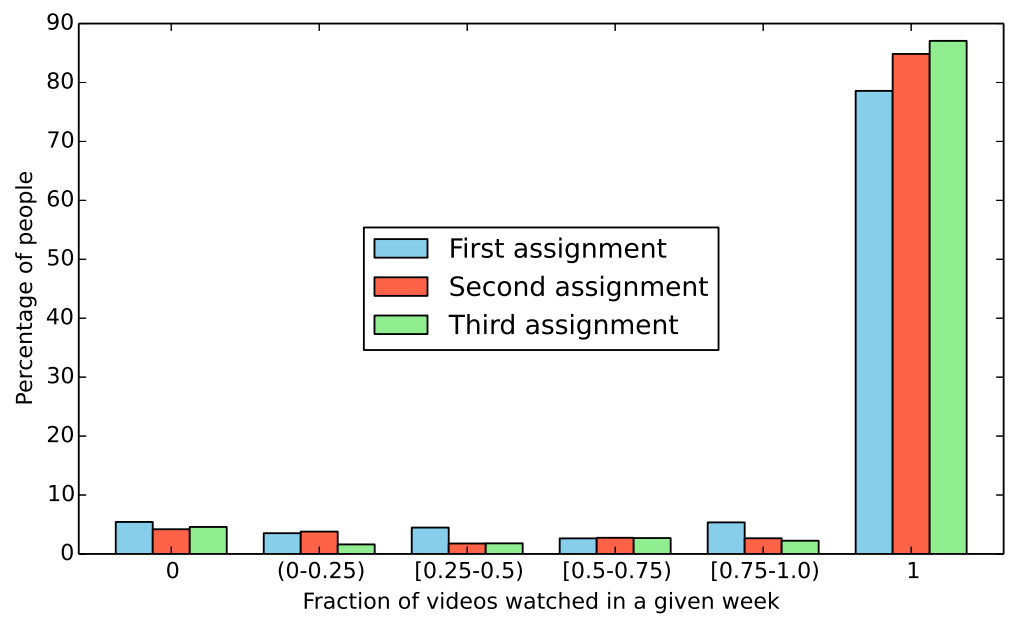

Figure 11: Distribution of the fraction of videos watched by learners in a given week for all three assignments. Here 0 means that they did not watch anything, and 1 means that they watched all available videos.

significant $(p=0.16)$.

\subsection{Testing the Reliability and the Validity of Peer Grading}

To address our fourth research question, i.e. the issue of validity and reliability of peer grading we randomly chose 300 essays from the second peer graded assignment and compared them with grades assigned by a group of undergraduate astronomy majors and minors, and also by a group of instructor graders. See the visualization in Figure 12. Writing prompt for this assignment is included in the Appendix A.

355 Except for having at their disposal a grading rubric (Appendix B) and correct answers to writing prompts (Appendix C) peer graders did not go through any calibration process nor they got any further guidelines for grading. The undergraduate graders went through a training set of 30 essays and we conducted three 60 minutes long training sessions where we discussed differences in our grades. Based on this training we constructed a more detailed rubric specific to the second peer graded assignment (Appendix D) which was used in the grading 


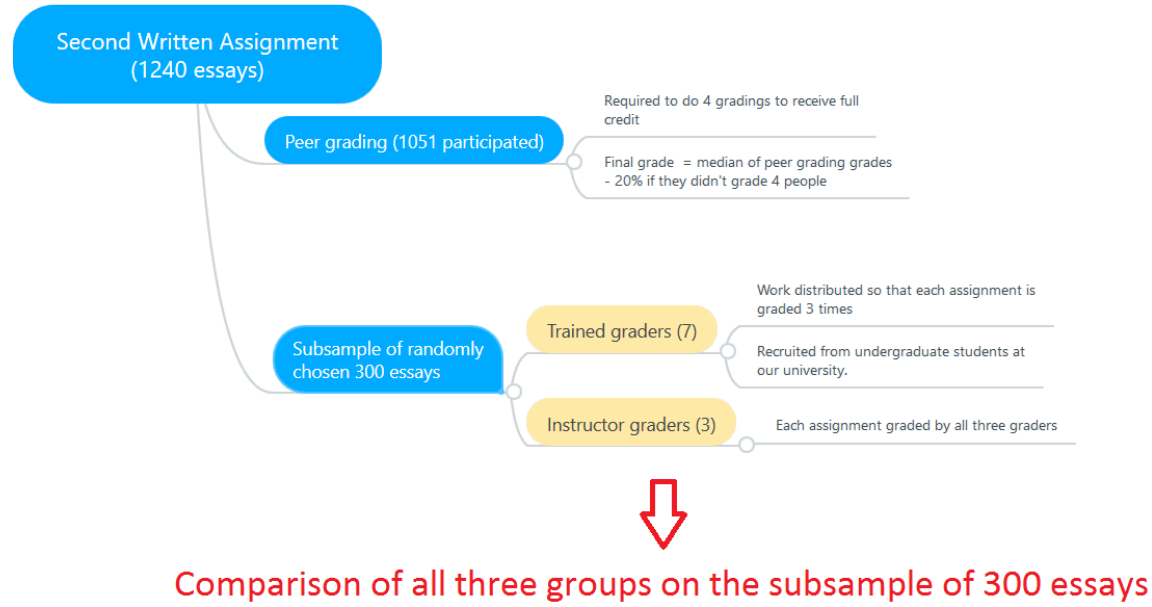

Figure 12: Visualization of the grading process used in our experiment.

experiment.

\section{Reliability of Peer Grading}

Comparison of the distributions of grades for all three groups of graders is presented in Figure 13. Peer graders tend to underestimate the top scoring submissions while overestimating the lowest scoring ones, compared to the instructor graders. This behavior is called "tendency to grade towards the mean" and it was also observed in a study by Piech et al. (2013). Our group of trained undergraduate graders also showed this effect.

Overall descriptive statistics for these three groups of graders were (Mean $=3.39$, St. dev. $=0.78)$ for peer graders, $($ Mean $=3.34$, St. dev. $=0.82)$ for undergraduate graders, and $($ Mean $=3.52$, St. dev. $=0.77)$ for instructor graders. A Kruskal-Wallis $\mathrm{H}$ test showed that distributions of assigned grades from these three groups of graders is statistically significantly different $\left(\chi^{2}(2)=\right.$ $36.36, p<0.001)$. From Dunn's post hoc test we found out that the group of instructor graders is different from the other two, i.e. instructor graders gave on average higher grades $(p<0.001)$. Difference between peer graders and trained undergraduate graders is not statistically significant $(p=0.25)$. 


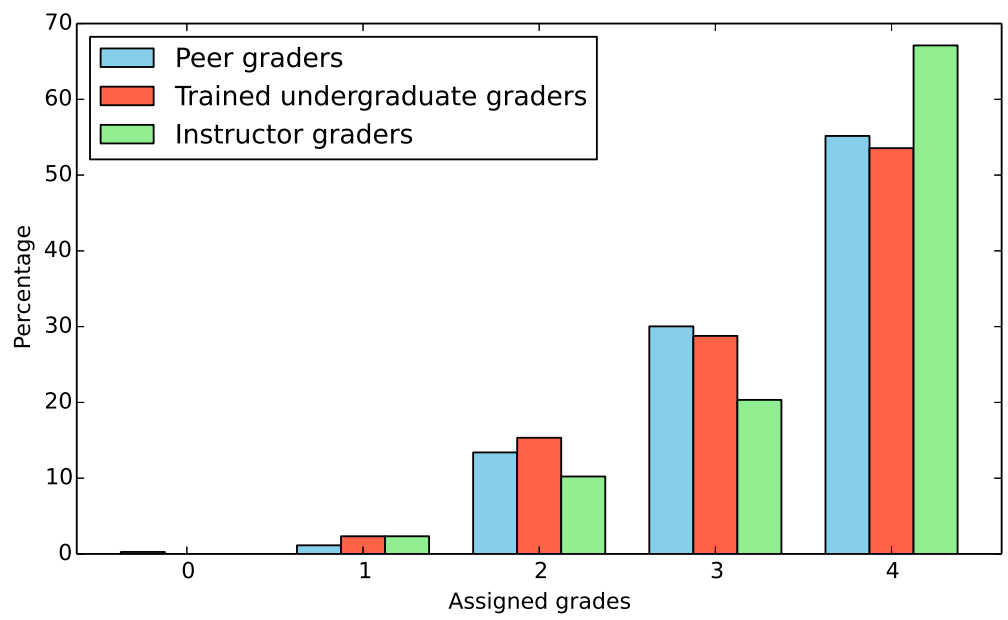

Figure 13: Comparison of distributions of scores for peer graders, trained undergraduate graders, and instructor graders on a sample of 300 essays for the second assignment.

As a measure of the reliability of different groups of graders we used the Intraclass Correlation Coefficient - ICC (Shrout \& Fleiss, 1979). For both peer graders and undergraduate graders we employed ICC(1) which is the case when each essay is rated by a different set of judges, randomly selected from a larger population. On the other hand for evaluation of the ICC of instructor graders we used ICC(3) because in this case each essay is rated by the same set of judges. Results for both single and average measures are presented in Table 2, Since some essays were graded by more than three peer graders, for the purposes of this analysis we considered only the first three grades. ICC as a criterion of reliability for peer graders in MOOC settings was also used in a previous study (Luo et al., 2014) and our results are very similar - only slightly higher with overlapping 95\% confidence intervals. Although the difference in grade distributions between peer graders and undergraduate graders turned out not statistically significant, we see a noticeable increase in reliability of undergraduate graders. As we expected the instructor graders turned out to be even more reliable, but far from being completely consistent.

\section{Validity of Peer Grading}




\begin{tabular}{|c||ccc|ccc|}
\hline \multicolumn{1}{|c||}{ Group of graders } & \multicolumn{3}{c|}{ Single Measures } & \multicolumn{3}{c|}{ Average Measures } \\
& $\mathrm{ICC}^{1}$ & Lower Bound & Upper Bound & $\mathrm{ICC}^{1}$ & Lower Bound & Upper Bound \\
\hline \hline Peer graders & .325 & .253 & .399 & .591 & .504 & .665 \\
Trained graders & .417 & .347 & .487 & .682 & .614 & .740 \\
Instructor graders & .631 & .575 & .684 & .837 & .802 & .866 \\
\hline
\end{tabular}

Table 2: Intraclass Correlation Coefficients (ICC) for all three groups of graders. Lower and upper bounds correspond to $95 \%$ confidence intervals. ${ }^{1} \operatorname{ICC}(1)$ for peer graders and undergraduate graders, ICC(3) for instructor graders.

Assuming we can consider a median of instructor grades as a "true scores" for any particular essay, we can also look on the validity of the peer grades. Table 3 lists all possible Pearson's correlation coefficients between the median grades assigned by all three groups of graders. The correlations are moderate to large with the strongest one being between instructor graders and undergraduate graders $(r=0.65, p<0.002)$ and the weakest one between instructor graders and peer graders $(r=0.49, p<0.002)$.

Finally, we present the distribution of differences between the "true scores" and grades assigned by peer graders (Mean $=-0.16$, St. dev. $=0.86)$ and undergraduate graders (Mean $=-0.21$, St. dev. $=0.77$ ) in Figure 14. We inquired whether these distributions are normal and although they turned out to be not skewed (skewness $=-0.005$ and -0.22 respectively) they are both leptokurtic (kurtosis (Fisher) $=1.12$ and 1.18 respectively) and therefore not normal. So for their comparison we used a Mann-Whitney U test instead of a t-test which showed that there is no statistically significant difference between these distributions $(U=499354, p=0.22)$.

\subsection{Relationships between grades and participation}

By comparing data presented in the section (5.3) on the participation level of individual users we discovered a couple of interesting trends. First, the learners who got better grades in the peer grading assignment generally worked harder on all aspects of the process (Figure 15). On average, they wrote longer essays, 


\begin{tabular}{|c||ccc|}
\hline & Peer graders & Trained graders & Instructor graders \\
\hline \hline Peer graders & 1 & 0.556 & 0.487 \\
Trained graders & & 1 & 0.647 \\
Instructor graders & & & 1 \\
\hline
\end{tabular}

Table 3: Correlations between median grades for all three groups of graders. All correlations are significant at 0.001 level.

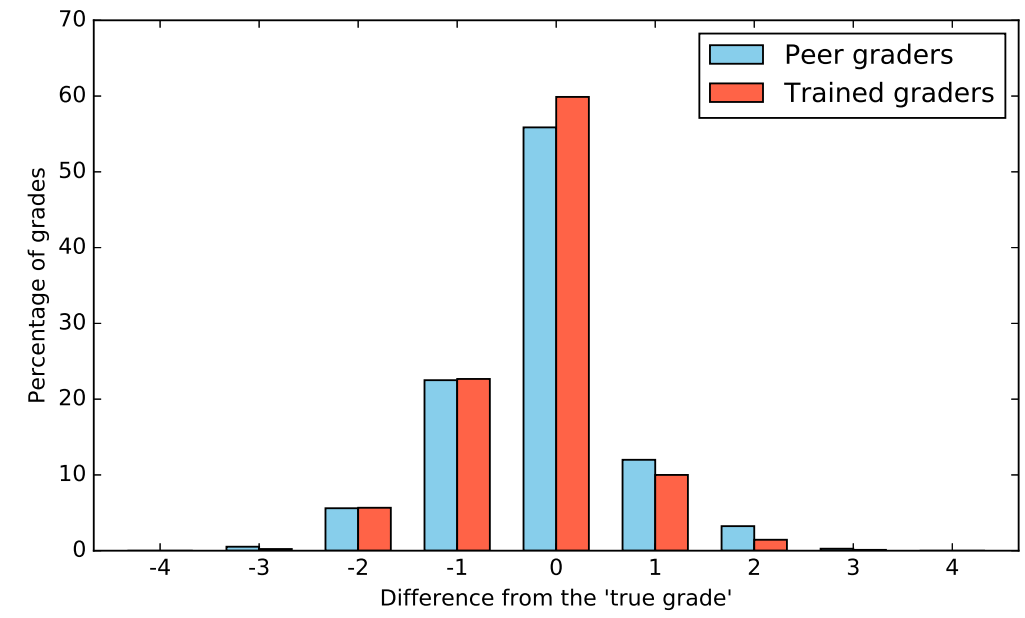

Figure 14: Distribution of differences between median instructor grades (or 'true scores') and both peer graders' and undergraduates' grades. 
did more reviews, spent longer time grading each essay, and watched more videos in a given week. All these relationships are linearly correlated and highly significant (Pearson's $r>0.9, p \leq 0.002$ ).

The correlation between grade and length of essay is probably not surprising. The learners who got the lowest grades wrote on average essays with 169 words, while the average for the learners with a perfect grade was 422 words (Figure 15a). The other differences are quite striking. For example, graders who got a top score from the assignment spent on average about 3.8 minutes grading each essay, while those who got 0 or 1 point graded for an average of about 0.8 or 1.7 minutes per essay respectively (Figure 15c). The impact of watching the instructional videos on assignment grade is also clearly manifested since learners who earned 0 or 1 point from the writing assignment watched on average only $51 \%$ or $68 \%$ of the videos in the week of the writing assignment, while those with the perfect scores watched on average $93 \%$ of them (Figure $15 \mathrm{~d}$ ).

Figure 16 relates the average times spent peer grading to the grade which the graders ultimately assigned and lengths of their essays. The data shows (Figure 16, left graph) that learners spent on average the longest time grading when they assigned a grade of 1 or 2 . This is not surprising because essays at both ends of the grade spectrum can be graded very quickly as opposed to those where the grade is not immediately clear. We also anticipated that average time spent grading per essay would be a monotonically increasing function of the essay lengths - learners who put more effort into their essays would also be more cautious graders. This is true up to essay lengths of $500-600$ words ${ }_{440}$ (Figure 16, right graph) where the distribution peaks and then unexpectedly drops. We speculate that learners who submitted essays with more than 600 words did not check the word limit and therefore were probably less invested in the writing assignment.

Finally we looked for a relationship between the median grades received 445 from the first writing assignment and the final course grades (Figure 17). This relationship turned out to be highly correlated $(r=0.98, p<0.001)$. This means that learners who scored higher in the first writing assignment on average also 

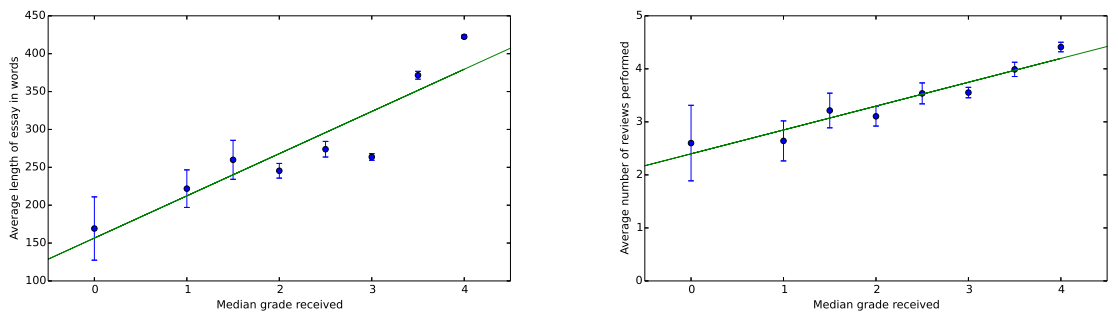

(a) Lengths of essays, $r=0.92, p=0.002$ (b) Number of reviews, $r=0.96, p<0.001$
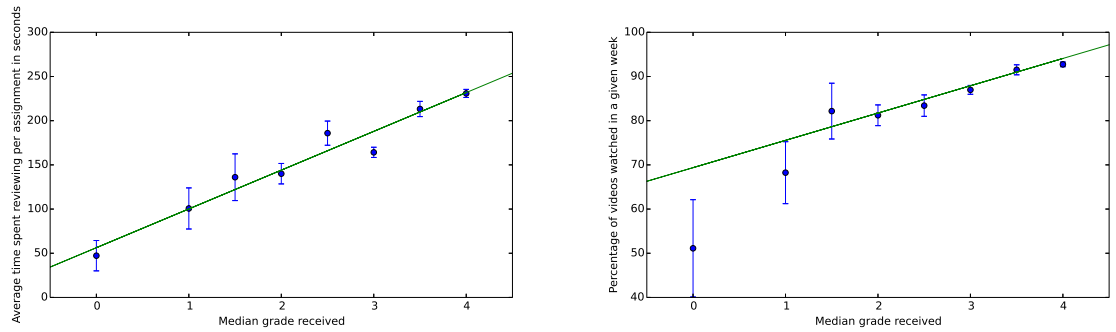

(c) Average grading times, $r=0.97, p<(d)$ Percentage of videos watched, $r=0.93$, 0.001

$$
p<0.001
$$

Figure 15: Relationships between median grades received from peer graders and other aspects of the peer grading process. Error bars indicate standard errors of the mean and lines represent linear fits.
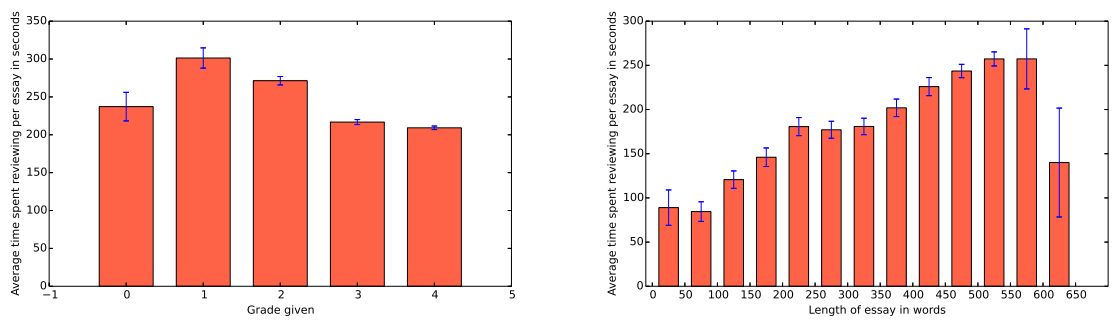

Figure 16: Trends between average time spent grading each assignment and grades given (left) or the length of essays (right). Error bars indicate standard errors of the mean. 


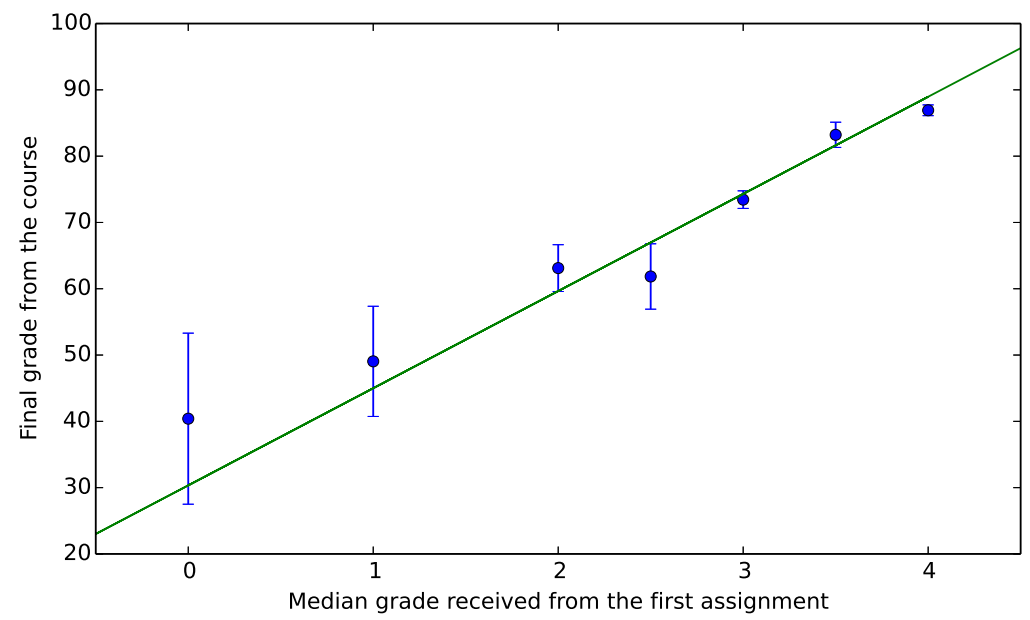

Figure 17: Relationship between median grades from the first writing assignment and final grades from the whole course $r=0.98, p<0.001$. Error bars indicate standard errors of the mean.

received higher final course grades. Of course, it can be argued that final course grade is trivially correlated with the grade from the first writing assignment because this grade is a part of the final grade. But in this case, according to our course description, it only amounts to $6.67 \%$ of the final grade meanwhile the change in the average final grade as seen in Figure 17 is almost 50\%.

\section{Summary of Findings}

Peer grading can play a major and pivotal role in a massive open online class. Through our investigation we found out that participation in the first peer graded assignment was the best predictor of completion for the course as whole, with as many as $81 \%$ of participants graduating and with a $89 \%$ graduation rate for peer graders.

From a demographic perspective, as compared to the general population of the course, learners from Asia were less likely to participate in the peer graded assignments and learners from Europe more likely. By looking at a subsample of learners who took an external entry survey, we found out that learners who 
participated in the peer graded assignments were more likely to be male, older, highly educated, and working in technical or professional fields, or tending to be educators or retired. The paper by Meek et al. (2016) reports very similar findings in a different field of a biomedical science MOOC. With the exception of the increased participation of educators this demographic shift is very similar to our findings about learners who graduated from the course (Impey et al., 2016). results for The level of previous astronomy knowlegde of our participant was generally very low, over $60 \%$ of learners had no previous academic experience with astronomy and over $80 \%$ of learners were mostly new or somewhat familiar with the subject.

In order to answer the third research question we compared all three peer graded assignments and we discovered that the first peer graded assignment was distinct from the other two in that learners on average graded more assignments, spent less time grading each essay and their submissions were shorter.

With a goal of evaluating validity and reliability of peer graders in mind we collected grading data from three distinct groups of graders; peer graders, trained undergraduate graders, and instructor graders. The distribution of grades for instructor graders was statistically significantly different from the other two, with higher average grades. Unlike in the study by Kulkarni et al. (2015) our peer graders turned out to be more harsh graders than instructors. There was no significant difference in grades between peer graders and undergraduate graders. The training of our undegraduate graders helped improve their reliability. Based on the Intraclass Correlation Coefficient the instructor graders were the most reliable with undergraduates following and peer graders the least reliable.

As far as validity of the grades is concerned the correlation between medians of instructor grades and medians of peer grades was moderate $(r=0.49)$ and the strongest correlation was between instructor graders and undergraduate graders $(r=0.65)$. There was no statistically significant difference for how the judgment of peer graders and undergraduate graders differed from the median instructor graders. 


\section{Conclusions and Future Research}

495

Similar studies of the peer grading have been conducted not only in traditional class settings (Cho et al., 2006), but for online classes (Bouzidi \& Jaillet, 2009) and MOOCs (Luo et al., 2014; Meek et al., 2016). The distinction we made in our experiment is that, unlike all these previous studies, we do not assume that the content expert is a perfectly reliable assessor, on the contrary, we were interested in the level of agreement or disagreement among our "expert" instructor graders. We were also curious about the effect of training on grades assigned by undergraduate graders.

Based on our investigations, we can conclude that the peer grading process as we used it in our course was not particularly reliable nor valid. The experiment with training our undergraduate graders helped improve their reliability, but it did not statistically significantly change their validity. That being said as we showed in section 5.5 results of the first peer grading assignment strongly correlate with overall course results (Figure 17), so despite the limited reliability and validity of the peer grading process it still on average identifies good learners. Moreover from correlations in Figure 15, we can see that learners who received higher grades from the peer grading generally tried harder in all aspects of the assignment. This is in agreement with findings of Comer et al. (2014) who also showed that better students engage and participate more across the board. On average they wrote statistically significantly longer essays, did more reviews, spent more time on grading, and watched more instructional videos. We would like to emphasize at this point that peer graders did not know their own grade before grading other learners, so there is no causal connection between their grade and the amount of effort they put into grading and yet once again the peer grading process awarded the learners who worked harder.

We would like to point out that our grading experiment revealed discrepancy between the grades assigned by our content experts. In our case content experts were instructor teaching assistants who are typically in charge of grading assignments for general education classes which can be considered on-campus 
equivalents of our MOOC course. With single measure ICC(3) of 0.63 and average measures ICC(3) of 0.84 there is certainly still a room for improving their reliability. Even instructors are to some extent susceptible to factors influencing the grading process as listed in Suen (2014). Therefore our results show that opinion of a single assessor may not be a good reference point for determining the overall validity of a peer grading process.

Finally, if we were to summarize what we think about a role of peer grading assignments in Massive Open Online Courses we came up with these conclusions:

1. Peer grading assignment implemented early enough in the course material serves as a successful predictor of completion for the course. At this point we cannot say if it selects out already motivated learners or if it contributes to their motivation.

2. Peer grading process and especially when feedback is required is beneficial for learners development on its own because it contributes to development of skills such as critical reflection, and sensible assessing (Liu \& Carless, 2006).

3. Although the peer grading has limits as far as validity and reliability are concerned, it still on average identifies learners who are more invested and who generally do better in the course.

These courses are finding an audience of motivated engaged learners as evidenced by the investment of many students in both writing responses and the peer review process. Future work should investigate differences in student peer review participation in courses like ours that draw a mostly lifelong learning audience compared to courses that have more career-development or professional improvement goals and audiences. We also want to understand more about how to both identify motivated learners, and to find ways to motivate learners. We are interested in trying to to increase student engagement in these online courses, and ultimately to encourage more students to complete. Although MOOCs are structurally similar to formal undergraduate level classes, the students are demographically different (Impey 2016) and the motivations for 
lifelong learners to take and participate in these courses are likely different than students in undergraduate classes. We want to understand more about these differences: what motivates MOOC learners to take MOOCs in the first place, what motivates them to finish, and how their motivations to learn differ from the motivations of students in formal classroom environments. These insights will guide us as we develop new learning and assessment strategies that can be effective for MOOC learners.

\section{Acknowledgments}

This work is supported by Howard Hughes Medical Institute grant number 52008138 for the project "Towards a Next Generation Online Science Course" and by the National Science Foundation under grant number 1244799. Any opinions, findings, and conclusions or recommendations expressed in this material are those of the authors and do not necessarily reflect the views of the National Science Foundation or the Howard Hughes Medical Institute. The authors are also grateful to Coursera for providing the data necessary for our analysis.

\section{Appendix A: Writing Prompts for the Peer Graded Assignments}

\section{- First Assignment}

Astronomers use telescopes to learn about the universe. Since the time of Galileo, optical telescopes have grown in aperture from a centimeter to ten meters, and astronomers have put telescopes in space and have used them to detect electromagnetic radiation at wavelengths not visible to the eye. In no more than 500 words, address the following questions about telescopes:

1. What are the advantages of large telescopes?

2. Why do astronomers want telescopes in space when putting them there is expensive? 
3. What are several examples of wavelength regions beyond the spectrum of visible light where astronomers can learn about the universe?

BEFORE YOU BEGIN! Plan ahead. Create a rough-outline so that you may better express your thoughts in a clear and ordered fashion. Be sure to use facts and relevant examples to reinforce and support your response.

BEFORE YOU SUBMIT! Be sure to unofficially self-assess based on the rubric.

\section{- Second Assignment}

Just twenty years have passed since the discovery of the first planet beyond the Solar System, and over 5000 exoplanets have been found. The limit on detection has reached Earthlike planets and some are habitable. Studying exoplanets recasts our view of our own Solar System. In no more than 500 words, address the following questions about planets near and far.

1. What are the two main, indirect methods for finding exoplanets?

2. Why is it so difficult to see exoplanets directly in an image?

3. What are some similarities or differences between our Solar System and new, distant planet systems?

BEFORE YOU BEGIN! Plan ahead. Create a rough-outline so that you may better express your thoughts in a clear and ordered fashion. Be sure to use facts and relevant examples to reinforce and support your response.

BEFORE YOU SUBMIT! Be sure to unofficially self-assess based on the rubric.

\section{- Third Assignment}

The understanding of how stars function to create elements and how they live and die is one of the greatest achievements of modern astronomy. The Sun is a typical star but some of the more extreme outcomes of evolution 
occur for massive stars. In no more than 500 words, address the following questions about star birth and death.

1. What is the source or cause of the Sun's light, and how do all the elements in the periodic table get produced?

2. What is the general process by which a large diffuse cloud of gas turns into a star and surrounding planets?

3. Name of the two end states of stars much more massive than the Sun and describe their physical properties?

BEFORE YOU BEGIN! Plan ahead. Create a rough-outline so that you may better express your thoughts in a clear and ordered fashion. Be sure to use facts and relevant examples to reinforce and support your response.

BEFORE YOU SUBMIT! Be sure to unofficially self-assess based on the rubric.

10. Appendix B: Writing Criteria and Rubric as given to MOOC learners

\section{Writing criteria:}

- The deadline for submission is 7 days after the Writing Assignment's initial opening.

- You are limited to a 500 word response.

- There is no set writing format we ask you to follow, but we do recommend that you carefully read the writing prompt and address exactly what is asked. A good way to ensure this is to restate the question(s). For example, should the writing prompt ask:

"What is the Scientific Method?"

You might begin by stating:

"The Scientific Method is..."

And then order your paragraphs/sections accordingly. 
- You might consider composing your response in a word processor (e.g., Microsoft Word) in order to better facilitate your writing. Once complete, simple copy and paste the text.

\section{How your writing will be evaluated:}

- Your response will be randomly assigned to four of your peers, who will evaluate your writing using the same 5 -point ( 0 to 4 ) rubric you see below. Your total grade will be the average of these four evaluations.

- All evaluations are due four days after the writing assignment deadline.

- Correct responses to each writing prompt question will be posted in The Week's Material immediately following the writing-assignment deadline.

\section{Evaluation Rubric:}

The goal of the writing is to correctly and concisely answer the questions based on the content in the video lectures. A five point scale (0-4 points) is used for quantitative grading. Each question is worth one point. The last possible point is for the quality and coherence of the response, or for adding extra information.

- 0 points - The writer does not correctly answer any of the questions.

- 1 point - The writer correctly answers one of the questions.

- 2 points - The writer correctly answers two of the questions.

- 3 points - The writer correctly answers three of the questions.

- 4 points - The writer correctly answers all three of the questions, expresses their thoughts clearly, and uses supporting facts and relevant examples.

Correct responses to each writing prompt question will be posted in The Week's

Material immediately following the writing-assignment deadline. 


\section{Appendix C: Posted Answers for Writing Assignment No. 2}

Just twenty years have passed since the discovery of the first planet beyond the Solar System, and over 5000 exoplanets have been found. The limit on detection has reached Earth-like planets and some are habitable. Studying exoplanets recasts our view of our own Solar System. In no more than 500 words, address the following questions about planets near and far.

1. What are the two main, indirect methods for finding exoplanets?

The two main methods are Doppler detection and transit (or eclipse) detection. In the Doppler method, the unseen planet reveals itself by tugging the parent star into a periodic Doppler shift. In the transit method, the planet crosses the face of the star and dims it slightly for a short time. In both methods, the signal repeats and the period of the orbit is measured.

2. Why is it so difficult to see exoplanets directly in an image?

Planets do not emit their own light. Therefore, they can only be seen in reflected light from the parent star. Depending on the planet size and distance from the star, that reflected light in many millions or even billions of times fainter than the nearby starlight, making it very difficult to detect.

3. What are some similarities or differences between our Solar System and new, distant planet systems? Our Solar System has small rocky planets close to the star and large, gas giant planet far away. Distant planets system are similar in that many of them have terrestrial or Earth-like planets, and many of them have giant planets too. Some distant planet systems are different because they have hot Jupiters close to their stars, and some have super-Earths which have no equivalent in our Solar System. One similarity and/or difference is sufficient.

\section{Appendix D: Detailed rubric for the 2nd assignment used in peer grading experiment}

- Question 1 
1. Because of the way the question is worded, students only need to list the two detection methods: Transit and Doppler. If they do, they should receive full credit and it should not count against them for the 4th point. They could also use terms like "Eclipse" or "Shadow" for the transit method, or "Wobble" or "Reflex" or "radial" or "RadialVelocity" for Doppler.

2. Students who only list one of these methods will receive no points. It's all or nothing.

3. Students can also lose the point for this question if they go into too much detail and explain something wrong, or include a third or fourth detection method.

4. Make sure they are not saying that the "wobble" or "reflex motion" can be directly observed. It cannot, and if they say it can be, it is wrong. It's indirectly observed by observing the Doppler shift of the star's spectrum.

5. There may be some confusion about what methods allow us to determine which properties of exoplanets (distance from the star, mass, orbital period, size, density), don't be too harsh here if they get these a bit mixed up.

- Question 2

1. As long as students say something about the relative brightness or size of planets compared with stars, they should receive full credit.

2. It is also acceptable to simply say that planets are dim, or are too close to their stars, or are far away from us without mentioning relative brightness or relative size, but it may not be sufficient to receive the 4th point for explanation.

3. It is not enough to simply say the star is bright or "because of the brightness of the star". Must say something about the planet, even that the planet is "dim" or "far away" are acceptable 
4. They could also say that it is easier to image planets in infrared light. They don't have to explain this to receive the point for this question.

5. Some students may say that "planets do not give off their own light" even though they do give off infrared light. Do not take off points if this is the only issue.

6. They do not have to say that the planet's light is reflected in order to receive credit.

\section{- Question 3}

1. In order to receive the point for this question, they only need to list one similarity or one difference.

2. They do not need to explicitly say it is a similarity or a difference to receive this point, but they do need to explain further in order to receive the 4 th point.

3. The best similarities would be things like: similar size planets in similar orbits, similar formation mechanisms, similar stars, planets in habitable zones, similar numbers of planets; could also include less directly relevant things like: planets orbit stars, orbits due to gravity. As long as it's technically correct, give them this first point, but unless they have a good explanation they should not receive the 4th point.

4. The best differences would be things like: Hot Jupiters close to their stars, more planets, different size planets, different kinds of stars, planets have more eccentric orbits; Again, as long as it's technically correct, they should receive the first point.

5. Some students may go into seemingly wild speculation about the chemical compositions of planets or their atmospheres, basically just ignore these things and see if they have something reasonable listed.

- 4th point

1. Students receive this if they have explained their answers clearly and do not have any obviously incorrect information 
2. Don't be too harsh about this requirement, but if there is anything that is really unclear, students will not receive this point.

- Other grading info

1. Do not take away points because something sounds like plagiarism. This is taken care of by Coursera. Only grade based on whether the information in the answer is correct.

2. Do not make judgements about a student's sources. Only focus on whether the answer is correct or incorrect.

3. Do not make assumptions about what a student does or does not seem to "understand". If the answer contains correct information, they should receive the first three points. If you think they don't understand, the only reason they shouldn't get the 4th point is because it's badly explained.

4. Do not take off points for grammar or spelling. Some students might not speak English as their first language. If the grammar is so bad it makes it difficult to understand their explanation that is the only reason to take off the 4 th point.

5. Even if they have great answers for the other 2 questions, if they are missing one answer, they cannot receive the 4 th point.

6. Be generous with the first 3 points even if answers are in the wrong order. Look around to see if the answers to the questions are in there, even if they aren't in the "right place". This disorganization is a good reason not to give the 4th point, but please go out of your way to look for the correct info somewhere in their answers.

\section{References}

Balfour, S. P. (2014). Asssessing writing in MOOCs: Automated essay scoring and Calibrated Peer Review ${ }^{\mathrm{TM}}$. Research $\mathscr{E}$ Practice in Assessment, 8, 4048. 
Bouzidi, L., \& Jaillet, A. (2009). Can online peer assessment be trusted? Educational Technology \& Society, 12(4), 257-268.

Cheng, W., \& Warren, M. (1997). Having second thoughts: student perceptions before and after a peer assessment exercise. Studies in Higher Education, 22, 233-239.

Cho, K., Schunn, C. D., \& Wilson, R. W. (2006). Validity and reliability of scaffolded peer assessment of writing from instructor and student perspectives. Journal of Educational Psychology, 98(4), 891-901. learning to write across the disciplines: Peer-to-peer writing in introductorylevel MOOCs. The International Review of Research in Open and Distributed Learning, 15.

Dochy, F., Segers, M., \& Sluijsmans, D. (1999). The use of self-, peer and coassessment in higher education: A review. Studies in Higher education, 24, $331-350$.

Falchikov, N. (1995). Peer feedback marking: developing peer assessment. Programmed Learning, 32, 175-187.

Falchikov, N., \& Goldfinch, J. (2000). Student peer assessment in higher education: A meta-analysis comparing peer and teacher marks. Review of Educational Research, 70(3), 287-322.

Furman, B., \& Robinson, W. (2003). Improving engineering report writing with Calibrated Peer Review ${ }^{\mathrm{TM}}$. In Proceedings of the 33rd Annual Frontiers in Education Conference. Piscataway, NJ: IEEE Digital Library.

Goldin, I. M., \& Ashley, K. D. (2011). Artificial Intelligence in Education: 15th International Conference. chapter Peering Inside Peer Review with Bayesian Models. (pp. 90-97). Berlin, Heidelberg: Springer Berlin Heidelberg. URL: http://dx.doi.org/10.1007/978-3-642-21869-9_14 doi $10.1007 / 978-3-642-21869-9 \backslash \_14$ 
Hollands, F. M., \& Tirthali, D. (2014). MOOCs: Expactations and reality, full report. Columbia University, NY: Center for Benefit-Cost Studies of Education, Teachers College.

Hunter, J. E. (1983). A causal analysis of cognitive ability, job knowledge, job performance, and supervisor ratings. Performance measurement and theory, 257, 266.

Impey, C. D., Hardegree-Ullman, K. K., Patikkal, A., Srinithan, A., Austin, C. L., Ganesan, N. K., \& Guvenen, B. C. (2013). A new online astronomy resource for education and outreach. Astronomy Education Review, 12. doi:10.3847/AER2012046.

815 Impey, C. D., Wenger, M. C., Formanek, M., \& Buxner, S. R. (2016). Bringing the universe to the world: Lessons learned from a massive open online class in astronomy. Communicating Astronomy with the Public, . Accepted, awaiting publication.

Jonsson, A., \& Svingby, G. (2007). The use of scoring rubrics: Reliability, validity and educational consequences. Educational research review, 2, 130144.

Kearns, L. R. (2012). Student assessment in online learning: Challenges and effective practices. Journal of Online Learning and Teaching, 8(3). URL: http://jolt.merlot.org/vol8no3/kearns_0912.htm

Keaten, J. A., \& Richardson, M. E. (1993). A field investigation of peer assessment as part of the student group grading process., .

Kolowich, S. (2013). The professors who make the MOOCs. The Chronicle of Higher Education, (p. 1).

Kulkarni, C., Wei, K. P., Le, H., Chia, D., Papadopoulos, K., Cheng, J., Koller, classes. In Design thinking research (pp. 131-168). Springer. 
Liu, N.-F., \& Carless, D. (2006). Peer feedback: the learning element of peer assessment. Teaching in Higher education, 11, 279-290.

Luo, H., Robinson, A. C., \& Park, J.-Y. (2014). Peer grading in a MOOC: Reliability, validity, and perceived effects. Journal of Asynchronous Learning Networks, 18(2).

Maas, A., Heather, C., Do, C. T., Brandman, R., Koller, D., \& Ng, A. (2014). Offering verified credentials in massive open online courses: Moocs and technology to advance learning and learning research (ubiquity symposium). Ubiquity, 2014, 2-11. URL: http://doi.acm.org/10.1145/2591684. doi $10.1145 / 2591684$

MacAndrew, P., \& Scanlon, E. (2013). Open learning at a distance: Lessons for struggling MOOCs. Science, 342, 1450-1451.

Meek, S. E., Blakemore, L., \& Marks, L. (2016). Is peer review an appropriate form of assessment in a MOOC? student participation and performance in formative peer review. Assessment $\&$ Evaluation in Higher Education, (pp. $1-14)$.

O'Toole, R. (2013). Pedagogical strategies and technologies for peer assessment in massively open online courses MOOCs, .

850 Panadero, E., \& Jonsson, A. (2013). The use of scoring rubrics for formative assessment purposes revisited: A review. Educational Research Review, 9, $129-144$.

Pappano, L. (2012). The year of the mooc. The New York Times, . URL: http://nyti.ms/18ndoGr.

${ }_{855}$ Piech, C., Huang, J., Chen, Z., Do, C., Ng, A., \& Koller, D. (2013). Tuned models of peer assessment in MOOCs. arXiv preprint arXiv:1307.2579, .

Pond, K., Ul-Haq, R., \& Wade, W. (1995). Peer review: a precursor to peer assessment. Programmed Learning, 32, 314-323. 
Reddy, Y. M., \& Andrade, H. (2010). A review of rubric use in higher education. Assessment ${ }^{3}$ evaluation in higher education, 35, 435-448.

Sadler, P. M., \& Good, E. (2006). The impact of self-and peer-grading on student learning. Educational assessment, 11, 1-31.

Sandeen, C. (2013). Assessment's place in the new MOOC world. Research $\mathscr{E}$ Practice in Assessment, 8, 5-12.

865 Shrout, P. E., \& Fleiss, J. L. (1979). Intraclass correlations: Uses in assessing rater reliability. Psychological Bulletin, 86(2), 420-428. doi:http://dx.doi.org/10.1037/0033-2909.86.2.420

Somervell, H. (1993). Issues in assessment, enterprise and higher education: The case for self-peer and collaborative assessment. Assessment and evaluation in Higher Education, 18, 221-233.

Suen, H. K. (2014). Peer assessment for massive open online courses (MOOCs). The International Review of Research in Open and Distance Learning, 15(3), $312-327$.

Topping, K. (1998). Peer assessment between students in colleges and universities. Review of educational Research, 68, 249-276.

Tseng, S.-C., \& Tsai, C.-C. (2007). On-line peer assessment and the role of the peer feedback: A study of high school computer course. Computers $\mathcal{E}$ Education, 49, 1161-1174.

Williams, E. (1992). Student attitudes towards approaches to learning and assessment. Assessment and evaluation in higher education, 17, 45-58. 